\title{
Analysis of a Single-Unit System with Weibull Failure and Repair densities Subject to Server Failure
}

\author{
${ }^{1}$ Ashish Kumar, ${ }^{2}$ Monika Saini \\ ${ }^{1}$ Department of Mathematics \& Statistics, Manipal University Jaipur, Jaipur-303007, Rajasthan, India. \\ *Corresponding Author: akbrk@ rediffmail.com
}

\begin{abstract}
The main concentration in the present paper is to analyze a single- unit system using the concepts of inspection, degradation, preventive maintenance, and server failure. A single repair facility inspect the original and degraded unit upon failure to check the feasibility of repair. The repair facility may fail during maintenance, repair and inspection and undergoes for treatment with Weibull probability density function. The unit after repair becomes degraded. Switch devices are perfect. All random variables like repair time, failure time, inspection time and treatment rate are independent and statistically independent. For the analysis of the system various measures of system effectiveness are obtained by using semi-Markov processes and regenerative point technique. With the help of numerical and graphical results for MTSF, availability and profit functions it is concluded that present system is more reliable, available and profitable, when random variables have less vale of shape parameters.
\end{abstract}

(Keywords: System, Availability, Inspection, Degradation and Preventive Maintenance)

\section{INTRODUCTION}

The configurations of modern industrial, mechanical, and communication systems becomes more and more complex with development of technology. The performance characteristics such as reliability, availability, mean time to system failure and profit incurred by the system depends more or less on the design of the system. A lot of researchers continuously try to develop new techniques of reliability improvement of such systems. Redundancy, replacement, priority and preventive maintenance are some such techniques that are used for reliability improvement. Out of these, redundancy is most commonly used technique to improve performance but it is not always possible to keep a stand by unit in spare due to some constraints like economic reasons. Further, the performance of a system can be improved by performing the preventive maintenance of the system after a pre-specified time. Several researcher like, Malik and Nandal (2010), Mahmoud and Moshref (2010), and Kumar et al. (2012) studied various reliability models by conducting preventive maintenance after a pre-specific time. Kumar and
Malik (2012) developed many stochastic models for a computer system using concept of preventive maintenance after maximum operation time and independent $\mathrm{h} / \mathrm{w}$ and $\mathrm{s} / \mathrm{w}$ failure. Malik and Bansal (2005), Malik (2008) and Pawar and Malik (2010) studied some reliability models by considering the assumption that the repair facility is never failed. But practically, this assumption seems unrealistic and he also meets a failure due to some accident or illness. In such a situation, he must go for some treatment and all repair and maintenance activities wait for his recovery. The general tendency in the existing literature is that repair of the failed unit is carried out by the server upon its failure but repair of a failed unit is not always possible. So, in such a situation first an inspection is performed to check the feasibility of repair and unit is replaced if it is not feasible for repair with some replacement time. Recently, Dhankhar and Malik (2011) studied a single-unit system with inspection time and perfect repair. But, repair of the unit many times depends on the skilled and unskilled repair man and extent of failure of unit. In such situations unit is not perfectly repaired and it becomes degraded. Barak 
et al. (2015) also developed reliability models under different set of assumptions. But, many mechanical and electrical system's failure and repair rates behaves arbitrarily. Osaki and Asakura (1970), Kapur and Kapoor (1974), Gupta et al. (2013) and Kishan and Jain(2014) suggested some reliability models for redundant systems in which all random variables are arbitrary distributed. Kumar and Saini (2014) discussed the cost-benefit analysis of a single-unit system with preventive maintenance and Weibull distribution. Kumar et al. (2015) analyzed a redundant system with the concepts of priority and Weibull repair and failure laws. The impact of abnormal weather conditions and inspection on repairable system has been analyzed by Barak and Barak (2016). Kumar et al. (2016) studied a single-unit system with degradation and maintenance. But in the literature of reliability modeling of industrial systems, no effort has been made for analyzing the performance of singleunit systems under preventive maintenance, degradation, inspection and server failure.

So, here an effort to obtain the reliability measures of system using these concepts. In all studies discussed above, reliability models for single -unit systems under different set of assumptions are developed. But, it's not always possible that repair and failure always shows the constant behavior. The performance of most of the mechanical, industrial and electrical systems varies with respect to passes of time. So, their repair and failure is not necessarily constant distributed but may behave as any arbitrary distribution. There are many distributions such as Weibull, normal, and lognormal distributions that are useful in analysing failure processes of standby systems. These distributions have hazard rate functions that are not constant over time, thus providing a necessary alternative to the exponential failure law. The most important probability distribution in reliability modelling is the Weibull distribution. The Weibull failure distribution may be used to model both increasing and decreasing failure rates. Suppose random variable $\mathrm{T}$ denotes the time to maximum operation time of an item/ device having Weibull distribution, and then its pdf is denoted by $f_{1}(t)=\theta \eta t^{\eta-1} \exp \left(-\theta t^{\eta}\right)$

$$
t \geq 0 \text { and } \theta, \eta>0 \text {. }
$$

It is characterized by a hazard rate function of the form $h(t)=\theta \eta t^{\eta-1}, t \geq 0$ and $\theta, \eta>0$ which is a power function. The function $\lambda(t)$ is increasing for $\eta>0, \theta>0$ and is decreasing for $\eta<0, \theta<0$. The reliability function is given by

$R(t)=\exp \left(-\theta t^{\eta}\right)$. Thus the failure free operating time of the system has a Weibull distribution with parameters $\theta$ and $\eta$. Here $\eta$ is referred to as the shape parameter. Its effect on the distribution can be seen for several different values. For $\eta<1$, the PDF is similar in the shape to the exponential, and for large value of $\eta(\eta \geq 3)$, the PDF is some-what symmetrical, like the normal distribution. For $1<\eta<3$, the density is skewed. If we put $\eta=1 \mathrm{in} \mathrm{pdf,} \mathrm{Weibull} \mathrm{distribution}$ reduces to Exponential distribution and if $\eta=2$, it reduces to Rayleigh distribution. Kumar and Saini (2014) analysed cost-benefit of a single-unit system under preventive maintenance and Weibull distribution for random variables.

Here we develop a reliability model for a single-unit system with server failure and Weibull distribution for repair and failure laws. The probability density function of all random variables followed by the time variables of the system are as follows:

Server failure /treatment rate :

$Z(t)=\mu \eta t^{\eta-1} \exp \left(-\mu t^{\eta}\right) / g(t)=\theta \eta t^{\eta-1} \exp \left(-\theta t^{\eta}\right)$

PM rate of original/degraded unit :

$f(t)=\alpha \eta t^{\eta-1} \exp \left(-\alpha t^{\eta}\right) / f_{1}(t)=\alpha_{1} \eta t^{\eta-1} \exp \left(-\alpha_{1} t^{\eta}\right)$

Inspection rate of original/degraded unit :

$h(t)=\beta \eta t^{\eta-1} \exp \left(-\beta t^{\eta}\right) / h_{1}(t)=\beta_{1} \eta t^{\eta-1} \exp \left(-\beta_{1} t^{\eta}\right)$

Repair rate of original/ degraded unit :

$m_{1}(t)=\gamma_{1} \eta t^{\eta-1} \exp \left(-\gamma_{1} t^{\eta}\right) / m_{1}(t)=\gamma_{1} \eta t^{\eta-1} \exp \left(-\gamma_{1} t^{\eta}\right)$

Failure rate of original/ degraded unit :

$X(t)=\lambda \eta t^{\eta-1} \exp \left(-\lambda t^{\eta}\right) / X_{1}(t)=\lambda_{1} \eta t^{\eta-1} \exp \left(-\lambda_{1} t^{\eta}\right)$

Maximum operation time :

$Y(t)=\alpha_{0} \eta t^{\eta-1} \exp \left(-\alpha_{0} t^{\eta}\right)$ with $t \geq 0$

The probability /cumulative density functions of direct transition time from regenerative state $i$ to $a$ regenerative state $\mathrm{j}$ or to a failed state $\mathrm{j}$ visiting state $\mathrm{k}$, $r$ once in $(0, t]$ have been denoted by $q_{i j . k r}(t) / Q_{i j . k r}(t)$. 
To improve the importance of the study, numerical results are obtained for a particular case for mean time to system failure, availability and profit function.

\section{SYSTEM DESCRIPTION}

A reliability model is developed in the present section by considering the concepts of inspection, preventive maintenance, degradation and server failure. The system may be any of the following states describes as follows:

State 0: Original unit is operative, repair facility is good and idle. The system is in upstate at $S_{0}$.

State 1: Original unit is under inspection, repair facility is good and busy in inspection of the failed unit. The system is in downstate state at $S_{1}$.

State 2: Original unit is under preventive maintenance, repair facility is good and busy in preventive maintenance of the unit. The system is in downstate state at $S_{2}$.

State 3: Original unit is waiting for preventive maintenance due to unavailability of repair facility, repair facility is under treatment. The system is in downstate state at $S_{3}$.

State 4: Original unit is waiting for inspection due to unavailability of repair facility, repair facility is under treatment. The system is in downstate state at $S_{4}$.

State 5: Original unit is under repair, repair facility is good and busy in repair of the failed unit. The system is in downstate state at $S_{5}$.

State 6: Original unit is waiting for repair due to unavailability of repair facility, repair facility is under treatment. The system is in downstate state at $S_{6}$.

State 7: Degraded unit is operative, repair facility is good and idle. The system is in upstate at $S_{7}$.

State 8: Degraded unit is under preventive maintenance, repair facility is good and busy in preventive maintenance of the unit. The system is in downstate state at $S_{8}$.

State 9: Degraded unit is under inspection, repair facility is good and busy in inspection of the failed unit. The system is in downstate state at $S_{9}$.

State 10: Degraded unit is under repair, repair facility is good and busy in repair of the failed unit. The system is in downstate state at $S_{10}$.

State 11: Degraded unit is waiting for inspection due to unavailability of repair facility, repair facility is under treatment. The system is in downstate state at $S_{11}$.

State 12: Degraded unit is waiting for preventive maintenance due to unavailability of repair facility, repair facility is under treatment. The system is in downstate state at $S_{12}$.

State 13: Degraded unit is waiting for repair due to unavailability of repair facility, repair facility is under treatment. The system is in downstate state at $S_{13}$.

Out of these, states $S_{0}$ and $S_{7}$ are operative states while all other are failed states. However, all the states of the system model are regenerative in nature.

\section{Transition Probabilities And Mean Sojourn Times}

Simple probabilistic considerations yield the following expressions for the non-zero elements $p_{i j}=Q_{i j}(\infty)=\int q_{i j}(t) d t$ as (1)

The transition probabilities at each state of the system model are derived as follows:

$\mathrm{p}_{01}=\int[$ Probability that system suffers by any failure and under inspection with good service facility during time $(t, t+d t)] d t$

$=\int_{0}^{\infty} X(t) \overline{Y(t)} d t=\int \lambda \eta t^{\eta-1} e^{-\lambda t^{\eta}} e^{-a_{0} \eta^{\eta}} d t=\lambda \eta \int t^{\eta-1} e^{-\left(\lambda+a_{0}\right) t^{\eta}} d t,=\frac{\lambda}{\alpha_{0}+\lambda}$

Similarly,

$\mathrm{p} 02=, \mathrm{p} 10=, \mathrm{p} 15=, \mathrm{p} 14=, \mathrm{p} 20=, \mathrm{p} 23=, \mathrm{p} 32=1=$ $\mathrm{p} 41, \mathrm{p} 56=, \mathrm{p} 57=, \mathrm{p} 65=1, \mathrm{p} 78=, \quad \mathrm{p} 79=, \mathrm{p} 87=$, p $8.12=, p 9.7=, p 9.10=, p 9.11=, p 10.13=, p 10.7=$, 
$\mathrm{p} 11.9=\mathrm{p} 12.8=\mathrm{p} 13.10=1$

It is easily verified that the sum of transition probabilities from one state to others is always equal to one.

The mean sojourn times $\left(\psi_{i}\right)$ is the state $S_{i}$ are

$$
\psi_{0}=\int_{0}^{\infty} \mathrm{e}^{-\left(\lambda+\alpha_{0}\right) \mathrm{t}^{\eta}} \mathrm{dt}=\frac{\Gamma(1+1 / \eta)}{\left(\lambda+\alpha_{0}\right)^{1 / \eta}},
$$

Similarly,

$$
\begin{array}{ll}
\psi_{1}=\frac{\Gamma(1+1 / \eta)}{(\beta+\mu)^{1 / \eta}}, & \psi_{2}=\frac{\Gamma(1+1 / \eta)}{(\alpha+\mu)^{1 / \eta}}, \\
\psi_{3}=\frac{\Gamma(1+1 / \eta)}{(\theta)^{1 / \eta}}, & \psi_{4}=\frac{\Gamma(1+1 / \eta)}{(\theta)^{1 / \eta}}, \mu_{5}=\frac{\Gamma\left(1+\frac{1}{\eta}\right)}{(\mu+\gamma)^{1 / \eta}} \\
\psi_{6}=\frac{\Gamma(1+1 / \eta)}{(\theta)^{1 / \eta}}, & \psi_{7}=\frac{\Gamma(1+1 / \eta)}{\left(\alpha_{0}+\lambda_{1}\right)^{1 / \eta}} \\
\psi_{8}=\frac{\Gamma(1+1 / \eta)}{\left(\alpha_{1}+\mu\right)^{1 / \eta}}, & \psi_{9}=\frac{\Gamma(1+1 / \eta)}{\left(\beta_{1}+\mu\right)^{1 / \eta}} \\
\psi_{10}=\frac{\Gamma(1+1 / \eta)}{\left(\gamma_{1}+\mu\right)^{1 / \eta}}, & \psi_{11}=\frac{\Gamma(1+1 / \eta)}{(\theta)^{1 / \eta}} \\
\psi_{12}=\frac{\Gamma(1+1 / \eta)}{(\theta)^{1 / \eta}}, & \psi_{13}=\frac{\Gamma(1+1 / \eta)}{(\theta)^{1 / \eta}}
\end{array}
$$

\section{Reliability And Mean Time To System Failure (Mtsf)}

Let $R_{i}(t)$ be the cdf of first passage time from the regenerative state to a failed state. Regarding the failed state as absorbing state, we have the following recursive relations for $R_{i}(t)$ :

$$
R_{0}(t)=Z_{0}(t) \quad \text { Where } \quad Z_{0}(t)=e^{-\left(\lambda+\alpha_{0}\right) t^{\eta}}
$$

Taking Laplace Steiltjes transformation of above relation (4) and solving for $R_{0}(s)$.We have

$\mathrm{R} *(\mathrm{~s})=\frac{1-R_{0}(s)}{s}$
The reliability of the system model can be obtained by taking Laplace inverse transform of (5).

The mean time to system failure (MTSF) is given by

$$
\mathrm{MTSF}=\lim _{s \rightarrow 0} \frac{1-R_{0}(s)}{s}=\psi_{0}
$$

\section{Steady State Availability}

Let $\mathrm{Ai}(\mathrm{t})$ be the probability that the system is in upstate at instant ' $\mathrm{t}$ ' given that the system entered regenerative state $\mathrm{i}$ at $\mathrm{t}=0$. The recursive relations for $\mathrm{Ai}(\mathrm{t})$ are given as

$$
A_{i}(t)=Z_{i}(t)+\sum_{j} q_{i, j}(t) \subseteq A_{j}(t)
$$

where $\mathrm{j}$ is any successive regenerative state $i$ to which the regenerative state $i$ can transit through $\mathrm{n}$ transitions. The probability that the system is initially up state up to time $\mathrm{t}$ without visiting to any other regenerative state $S_{i} \in E$, we have

$$
Z_{0}(t)=e^{-\left(\alpha_{0}+\lambda\right) t^{\eta}}, \text { and } Z_{7}(t)=e^{-\left(\alpha_{0}+\lambda_{1}\right) t^{\eta}}
$$

Taking LT of above relations given in equation (7) and solving for $A_{0}^{*}(s)$ steady state availability is given by

$$
A_{0}(\infty)=\lim _{s \rightarrow 0} s A_{0}^{*}(s) \quad=\frac{N_{2}}{D_{2}} \quad, \quad \text { where }
$$

$$
\begin{aligned}
N_{2}= & \left(Z_{0}(t)\left(1-p_{14} p_{41}\right)\left(1-p_{32} p_{23}\right)\left(1-p_{56} p_{65}\right)\right)\left(1-p_{8.12} p_{12.8}\right)\left(1-p_{11.9} p_{9.11}\right)\left(1-p_{10.13} p_{13.10}\right) \\
& -p_{79} p_{97}\left(1-p_{8.12} p_{12.8}\right)\left(1-p_{10.13} p_{13.10}\right)-p_{78} p_{87}\left(1-p_{11.9} p_{9.11}\right)\left(1-p_{10.13} p_{13.10}\right)-p_{10.7} p_{79} \\
& \left.p_{9.10}\left(1-p_{8.12} p_{12.8}\right)\right)+Z_{7}(t) p_{0.1} p_{57} p_{15}\left(1-p_{13.10} p_{10.13}\right)\left(1-p_{32} p_{23}\right)\left(1-p_{9.11} p_{11.1}\right)\left(1-p_{8.12} p_{12.2}\right)
\end{aligned}
$$

\section{Busy Period Analysis For Repairman}

Recurrence relations of $B_{i}(t)$ are as follows:

$$
B_{i}(t)=Z_{i}(t)+\sum_{j} q_{i, j}(t) \subseteq B_{j}(t)
$$

$\mathrm{Z}_{\mathrm{i}}(\mathrm{t})$ be the probability that the server is busy in state $\mathrm{S}_{\mathrm{i}}$ up to time $t$ without making any transition to any other regenerative state or returning to the same via one or 
more non-regenerative states and so $Z_{1}(t)=e^{-(\beta+\mu) t^{\eta}}$, $Z_{2}(t)=e^{-(\alpha+\mu) t^{\eta}}, Z_{5}(t)=e^{-(\gamma+\mu) t^{\eta}}, \quad Z_{8}(t)=e^{-\left(\alpha_{1}+\mu\right) t^{\eta}}$, $Z_{9}(t)=e^{-\left(\beta_{1}+\mu\right) t^{\eta}}$ and $Z_{10}(t)=e^{-\left(\gamma_{1}+\mu\right) t^{\eta}}$. By taking LT of (10) and solving for $B_{0}^{*}(s)$. The busy period of the

$$
\begin{aligned}
& N_{3}=\left(W_{1}(t) p_{011}\left(1-p_{32} p_{23}\right)\left(1-p_{56} p_{65}\right)+W_{2}(t) p_{02}\left(1-p_{14} p_{41}\right)\left(1-p_{56} p_{65}\right)+W_{5}(t) p_{15} p_{01}\right. \\
& \left.\left(1-p_{32} p_{23}\right)\right)\left(\left(1-p_{8.12} p_{12.8}\right)\left(1-p_{9.11} p_{11.9}\right)\left(1-p_{10.13} p_{13.10}\right)-p_{78} p_{87}\left(1-p_{9.11} p_{11.9}\right)\right. \\
& \left.\left(1-p_{10.13} p_{13.10}\right)-p_{79} p_{97}\left(1-p_{8.12} p_{12.8}\right)\left(1-p_{10.13} p_{13.10}\right)-p_{79} p_{9.10} p_{10.7}\left(1-p_{8.12} p_{12.8}\right)\right)+ \\
& p_{57} p_{75} p_{011}\left(1-p_{23} p_{32}\right)\left(\left(1-p_{9.11} p_{11.9}\right)\left(1-p_{10.13} p_{13.10}\right) p_{78} W_{8}+\left(1-p_{8.12} p_{12.2}\right)\left(1-p_{10.13} p_{13.10}\right)\right. \\
& \left.\left.\left.\left.p_{79} W_{9}+p_{79} W_{10} p_{9.10}\left(1-p_{8.12} p_{12.8}\right)\right) p_{31.12} p_{13.10}\right)-p_{14.9} p_{31.12} p_{23.6}\right)\right) \\
& \qquad B_{0}^{R}=\lim _{s \rightarrow 0} s B_{0}^{* R}(s)=\frac{N_{3}^{R}}{D_{2}} \text {, where }
\end{aligned}
$$

And $\mathrm{D}_{2}$ is already mentioned in previous section.

\section{PROFIT ANALYSIS}

The profit incurred to the system model in steady state can be obtained as

$$
P=K_{0} A_{0}-K_{1} B_{0}
$$

$\mathrm{K}_{0}=$ Revenue per unit up-time of the system

$\mathrm{K}_{1}=$ Cost per unit time for which server is busy in repair activities.

\section{CASE STUDIES WITH DISCUSSIONS}

(i) When shape parameter $\eta=0.5$ then treatment rate/ preventive maintenance rate of original unit/ inspection rate of original unit/inspection rate of degraded unit/repair rate of original unit/ preventive maintenance rate of degraded unit/ repair rate of degraded unit/ failure rate of original unit/ failure rate of degraded unit/maximum operation time of units, and server failure time distributions reduces to:

$$
\begin{aligned}
& g(t)=\frac{\theta}{2 \sqrt{t}} e^{-\theta \sqrt{t}}, f(t)=\frac{\alpha}{2 \sqrt{t}} e^{-\alpha \sqrt{t}}, h(t)=\frac{\beta}{2 \sqrt{t}} e^{-\beta \sqrt{t}}, h_{1}(t)=\frac{\beta_{1}}{2 \sqrt{t}} e^{-\beta_{1} \sqrt{t}}, m(t)=\frac{\gamma}{2 \sqrt{t}} e^{-\gamma \sqrt{t}}, \\
& f_{1}(t)=\frac{\alpha_{1}}{2 \sqrt{t}} e^{-\alpha_{1} \sqrt{t}}, m_{1}(t)=\frac{\gamma_{1}}{2 \sqrt{t}} e^{-\gamma_{1} \sqrt{t}}, X(t)=\frac{\lambda}{2 \sqrt{t}} e^{-\lambda \sqrt{t}}, X_{1}(t)=\frac{\lambda_{1}}{2 \sqrt{t}} e^{-\lambda_{1} \sqrt{t}}, Y(t)=\frac{\alpha_{0}}{2 \sqrt{t}} e^{-\alpha_{0} \sqrt{t}}, \\
& Z(t)=\frac{\mu}{2 \sqrt{t}} e^{-\mu \sqrt{t}} ; \text { where } t \geq 0 \text { and } \eta, \alpha, \theta, \alpha_{1}, \mu, \beta, \beta_{1}, \gamma_{1}, \gamma, \lambda, \lambda_{1}, \alpha_{0}>0
\end{aligned}
$$

The mean sojourn times $\left(\psi_{i}\right)$ in the state $S_{\mathrm{i}}$ after using (12) reduces to:

$$
\begin{aligned}
& \psi_{0}=\frac{2}{\left(\lambda+\alpha_{0}\right)^{2}}, \psi_{1}=\frac{2}{(\beta+\mu)^{2}}, \psi_{2}=\frac{2}{(\alpha+\mu)^{2}}, \\
& \psi_{3}=\frac{2}{(\theta)^{2}}, \psi_{4}=\frac{2}{(\theta)^{2}}, \mu_{5}=\frac{2}{(\mu+\gamma)^{2}}, \\
& \psi_{6}=\frac{2}{(\theta)^{2}}, \psi_{7}=\frac{2}{\left(\alpha_{0}+\lambda_{1}\right)^{2}}, \psi_{8}=\frac{2}{\left(\alpha_{1}+\mu\right)^{2}}, \\
& \psi_{9}=\frac{2}{\left(\beta_{1}+\mu\right)^{2}}, \psi_{10}=\frac{2}{\left(\gamma_{1}+\mu\right)^{2}}, \\
& \frac{2}{(\theta)^{2}}, \psi_{12}=\frac{2}{(\theta)^{2}}, \psi_{13}=\frac{2}{(\theta)^{2}}
\end{aligned}
$$

ii) When shape parameter $\eta=1$ then treatment rate/ preventive maintenance rate of original unit/ inspection rate of original unit/inspection rate of degraded unit/repair rate of original unit/ preventive maintenance rate of degraded unit/ repair rate of degraded unit/ failure rate of original unit/ failure rate of degraded unit/maximum operation time of units, and server failure time distributions reduces to:

$$
\begin{aligned}
& g(t)=\theta e^{-\theta t}, f(t)=\alpha e^{-\alpha t}, h(t)=\beta e^{-\beta t}, h_{1}(t)=\beta_{1} e^{-\beta_{1} t}, m(t)=\gamma e^{-\gamma t}, f_{1}(t)=\alpha_{1} e^{-\alpha_{1} t^{t}}, \\
& m_{1}(t)=\gamma_{1} e^{-\gamma_{1} t}, X(t)=\lambda e^{-\lambda t}, X_{1}(t)=\lambda_{1} e^{-\lambda_{1} t}, Y(t)=\alpha_{0} e^{-\alpha_{0} t}, Z(t)=\mu e^{-\mu t} ; \quad \text { where } t \geq 0 \\
& \text { and } \eta, \alpha, \theta, \alpha_{1}, \mu, \beta, \beta_{1}, \gamma_{1}, \gamma, \lambda, \lambda_{1}, \alpha_{0}>0
\end{aligned}
$$

The mean sojourn times $\left(\psi_{i}\right)$ in the state $S_{\mathrm{i}}$ after using (14) reduces to:

$\psi_{0} \frac{1}{\left(\lambda+\alpha_{0}\right)} \psi_{1} \frac{1}{(\beta+\mu)} \psi_{2}=\frac{1}{(\alpha+\mu)} \psi_{3} \frac{1}{(\theta)}$
$\psi_{4} \frac{1}{(\theta)} \mu_{5}=\frac{1}{(\mu+\gamma)} \psi_{6}=\frac{1}{(\theta)} \psi_{7}=\frac{1}{\left(\alpha_{0}+\lambda_{1}\right)}$ 
$\psi_{8}=\frac{1}{\left(\alpha_{1}+\mu\right)} \psi_{9}=\frac{1}{\left(\beta_{1}+\mu\right)} \psi_{10}=\frac{1}{\left(\gamma_{1}+\mu\right)}$

$\psi_{11} \frac{1}{(\theta)} \psi_{12} \frac{1}{(\theta)} \psi_{13} \frac{1}{(\theta)}$

(iii) When shape parameter $\eta=2$ then treatment rate/ preventive maintenance rate of original unit/ inspection rate of original unit/inspection rate of degraded unit/repair rate of original unit/ preventive maintenance rate of degraded unit/ repair rate of degraded unit/ failure rate of original unit/ failure rate of degraded unit/maximum operation time of units, and server failure time distributions reduces to:

$$
\begin{aligned}
& g(t)=2 \theta t e^{-\theta t^{2}}, f(t)=2 \alpha t e^{-\alpha t^{2}}, h(t)=2 \beta t e^{-\beta t_{1}{ }^{2}}, h_{1}(t)=2 \beta_{1} t e^{-\beta_{1} t^{2}}, m(t)=2 \gamma t e^{-\gamma t^{2}}, \\
& f_{1}(t)=2 \alpha_{1} t e^{-\alpha_{1}{ }^{2}}, m_{1}(t)=2 \gamma_{1} t e^{-\gamma \gamma_{1}{ }^{2}}, X(t)=2 \lambda t e^{-\lambda t^{2}}, X_{1}(t)=2 \lambda_{1} t e^{-\lambda_{1} t^{2}}, Y(t)=2 \alpha_{0} t e^{-\alpha_{0} t^{2}}, \\
& Z(t)=2 \mu t t e^{-\mu t^{2}} \text {; where } t \geq 0 \text { and } \eta, \alpha, \theta, \alpha_{1}, \mu, \beta, \beta_{1}, \gamma_{1}, \gamma, \lambda, \lambda_{1}, \alpha_{0}>0
\end{aligned}
$$

The mean sojourn times $\left(\psi_{i}\right)$ in the state $S_{\mathrm{i}}$ after using (15) reduces to:

$$
\begin{aligned}
& \psi_{0}=\frac{1}{2} \sqrt{\frac{\pi}{\left(\lambda+\alpha_{0}\right)}}, \psi_{1}=\frac{1}{2} \sqrt{\frac{\pi}{(\beta+\mu)}}, \\
& \psi_{2}=\frac{1}{2} \sqrt{\frac{\pi}{(\alpha+\mu)}}, \psi_{3}=\frac{1}{2} \sqrt{\frac{\pi}{(\theta)}}, \psi_{4}=\frac{1}{2} \sqrt{\frac{\pi}{(\theta)}}, \\
& \mu_{5}=\frac{1}{2} \sqrt{\frac{\pi}{(\mu+\gamma)}}, \psi_{6}=\frac{1}{2} \sqrt{\frac{\pi}{(\theta)}}, \psi_{7}=\frac{1}{2} \sqrt{\frac{\pi}{\left(\alpha_{0}+\lambda_{1}\right)}}, \\
& \psi_{8}=\frac{1}{2} \sqrt{\frac{\pi}{\left(\alpha_{1}+\mu\right)}}, \psi_{9}=\frac{1}{2} \sqrt{\frac{\pi}{\left(\beta_{1}+\mu\right)}}, \\
& \psi_{10}=\frac{1}{2} \sqrt{\frac{\pi}{\left(\gamma_{1}+\mu\right)}}, \psi_{11}=\frac{1}{2} \sqrt{\frac{\pi}{(\theta)}}, \psi_{12}=\frac{1}{2} \sqrt{\frac{\pi}{(\theta)}}, \\
& \psi_{13}=\frac{1}{2} \sqrt{\frac{\pi}{(\theta)}}
\end{aligned}
$$

\section{NUMERICAL RESULTS}

Table 1: MTSF vs. Failure Rate $(\lambda)$

\begin{tabular}{lllllll}
\hline$\lambda$ & $\eta=0$. & $\eta=0$. & $\eta=1$, & $\eta=1$, & $\eta=2$, & $\eta=2$, \\
& 5, & 5, & $\theta=0.0$ & $\theta=0.1$ & $\theta=0.0$ & $\theta=0$. \\
& $\theta=0.0$ & $\theta=0$. & 5 & & 5 & 1 \\
& 5 & 1 & & & & \\
\hline 0.0 & 1996 & 1996 & 99.90 & 99.90 & 8.857 & 8.857 \\
1 & 0 & 0 & 01 & 01 & 8 & 8 \\
0.0 & 4995 & 4995 & 49.97 & 49.97 & 6.265 & 6.265 \\
2 & 2221 & 2221 & 50 & 50 & 0 & 0 \\
0.0 & 1249 & 1249 & 33.32 & 33.32 & 5.115 & 5.115 \\
3 & 800 & 800 & 22 & 22 & 8 & 8 \\
0.0 & 555 & 555 & 24.99 & 24.99 & 4.430 & 4.430 \\
4 & 408 & 408 & 38 & 38 & 6 & 6 \\
0.0 & 312 & 312 & 19.99 & 19.99 & 3.962 & 3.962 \\
5 & 247 & 247 & 60 & 60 & 9 & 9 \\
0.0 & 200 & 200 & 16.66 & 16.66 & 3.617 & 3.617 \\
6 & & & 39 & 39 & 7 & 7 \\
0.0 & & & 14.28 & 14.28 & 3.349 & 3.349 \\
7 & & & 37 & 37 & 4 & 4 \\
0.0 & & & 12.49 & 12.49 & 3.133 & 3.133 \\
8 & & & 84 & 84 & 1 & 1 \\
0.0 & & & 11.10 & 11.10 & 2.953 & 2.953 \\
9 & & & 99 & 99 & 9 & 9 \\
0.1 & & & 9.999 & 9.999 & 2.802 & 2.802 \\
0 & & & 0 & 0 & 4 & 4 \\
\hline
\end{tabular}

Table 2: Availability vs. Failure Rate $(\lambda)$

\begin{tabular}{lllllll}
\hline$\lambda$ & $\eta=0.5$ & $\eta=0.5$ & $\eta=1$, & $\eta=1$, & $\eta=2$, & $\eta=2$, \\
&, &, & $\theta=0.0$ & $\theta=0.1$ & $\theta=0.0$ & $\theta=0.1$ \\
& $\theta=0.0$ & $\theta=0.1$ & 5 & & 5 & \\
& 5 & & & & & \\
\hline 0.0 & 0.942 & 0.942 & 0.856 & 0.856 & 0.799 & 0.800 \\
1 & 3 & 3 & 0 & 3 & 7 & 8 \\
0.0 & 0.940 & 0.940 & 0.842 & 0.842 & 0.776 & 0.777 \\
2 & 0 & 0 & 3 & 6 & 7 & 8 \\
0.0 & 0.939 & 0.939 & 0.837 & 0.838 & 0.767 & 0.768 \\
3 & 5 & 5 & 8 & 1 & 0 & 0 \\
0.0 & 0.939 & 0.939 & 0.835 & 0.835 & 0.761 & 0.762 \\
4 & 4 & 4 & 6 & 9 & 3 & 3 \\
0.0 & 0.939 & 0.939 & 0.834 & 0.834 & 0.757 & 0.758 \\
5 & 3 & 3 & 3 & 6 & 4 & 5 \\
0.0 & 0.939 & 0.939 & 0.833 & 0.833 & 0.754 & 0.755 \\
6 & 3 & 3 & 4 & 7 & 6 & 6 \\
0.0 & 0.939 & 0.939 & 0.832 & 0.833 & 0.752 & 0.753 \\
7 & 3 & 3 & 8 & 1 & 5 & 5 \\
0.0 & 0.939 & 0.939 & 0.832 & 0.832 & 0.750 & 0.751 \\
8 & 2 & 3 & 3 & 6 & 7 & 7 \\
0.0 & 0.939 & 0.939 & 0.832 & 0.832 & 0.749 & 0.750 \\
9 & 2 & 2 & 0 & 2 & 3 & 3 \\
0.1 & 0.939 & 0.939 & 0.831 & 0.831 & 0.748 & 0.749 \\
0 & 2 & 2 & 7 & 9 & 1 & 1 \\
\hline
\end{tabular}


Table 3: Profit vs. Failure Rate $(\lambda)$

\begin{tabular}{lllllll}
\hline$\lambda$ & $\eta=0.5$ & $\eta=0.5$ & $\eta=1$, & $\eta=1$, & $\eta=2$, & $\eta=2$, \\
&, &, & $\theta=0.0$ & $\theta=0.1$ & $\theta=0.0$ & $\theta=0.1$ \\
& 5 & & & & 5 & \\
\hline 0. & 471.1 & 471.1 & 427.9 & 428.0 & 397.3 & 397.9 \\
01 & 281 & 331 & 201 & 706 & 370 & 044 \\
0. & 469.9 & 469.9 & 421.0 & 421.2 & 385.9 & 386.4 \\
02 & 786 & 836 & 689 & 146 & 281 & 635 \\
0. & 469.7 & 469.7 & 418.8 & 418.9 & 381.0 & 381.6 \\
03 & 656 & 705 & 338 & 780 & 869 & 090 \\
0. & 469.6 & 469.6 & 417.7 & 417.8 & 378.2 & 378.7 \\
04 & 908 & 958 & 251 & 686 & 601 & 744 \\
0. & 469.6 & 469.6 & 417.0 & 417.2 & 376.3 & 376.8 \\
05 & 561 & 611 & 627 & 057 & 556 & 647 \\
0. & 469.6 & 469.6 & 416.6 & 416.7 & 374.9 & 375.4 \\
06 & 372 & 422 & 223 & 650 & 623 & 677 \\
0. & 469.6 & 469.6 & 416.3 & 416.4 & 373.8 & 374.3 \\
07 & 258 & 307 & 083 & 508 & 868 & 893 \\
0. & 469.6 & 469.6 & 416.0 & 416.2 & 373.0 & 373.5 \\
08 & 184 & 233 & 731 & 154 & 244 & 246 \\
0. & 469.6 & 469.6 & 415.8 & 416.0 & 372.3 & 372.8 \\
09 & 132 & 182 & 904 & 325 & 132 & 115 \\
0. & 469.6 & 469.6 & 415.7 & 415.8 & 371.7 & 372.2 \\
10 & 096 & 145 & 443 & 864 & 137 & 104 \\
& & & & & & \\
\hline
\end{tabular}

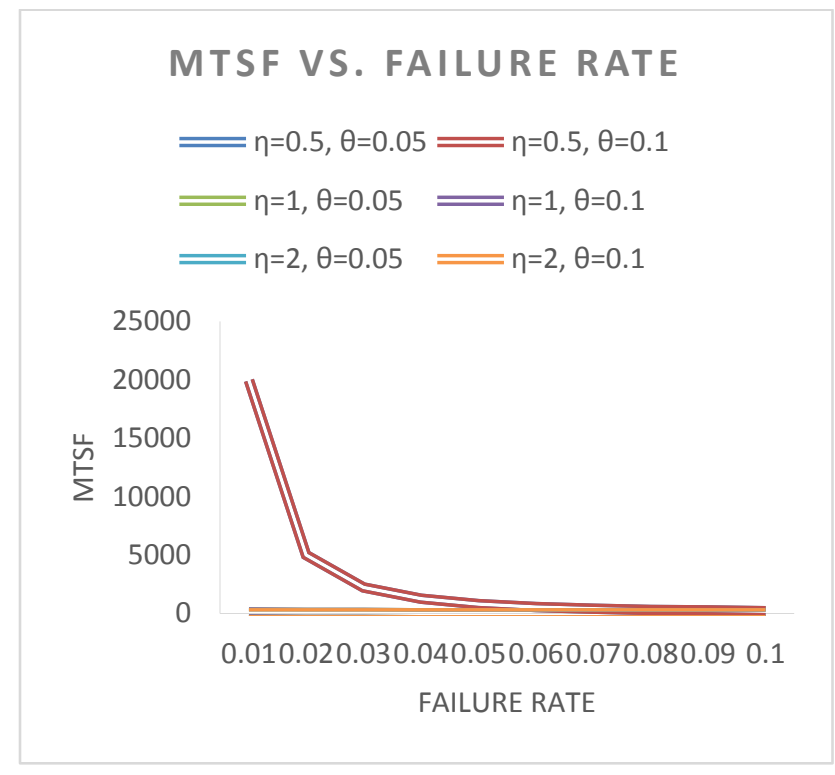

Figure 1: MTSF vs. Failure Rate

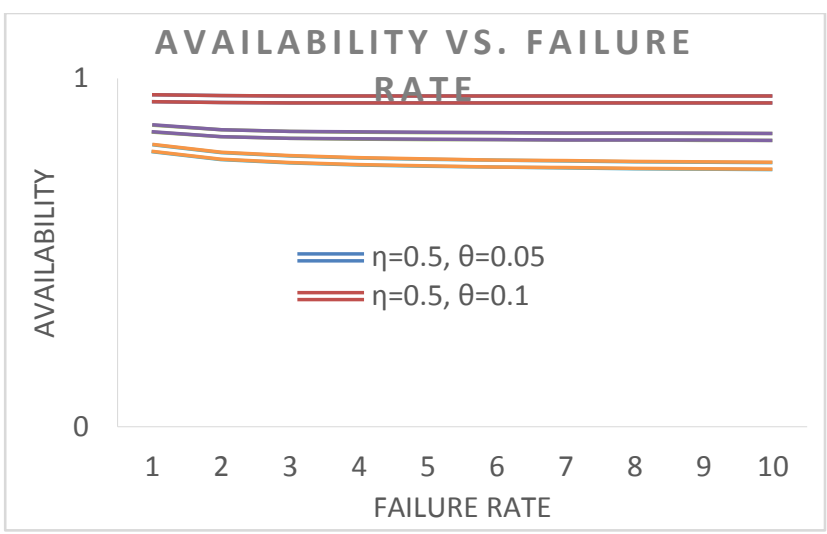

Figure 2: Availability vs. Failure Rate

\section{CONCLUSION}

In the present paper, various reliability measures such as mean time to system failure, availability, and profit function are derived for different values of shape parameters for a single-unit system with Weibull distributed random variables. Tables 1-3, highlights the behaviors of reliability measures with respect to failure rate $(\lambda)$ and different values of server treatment rate $(\theta=0.05 \& \theta=0.1)$. The values of other parameters are kept fixes here as $\alpha=0.5, \beta=0.004, \beta_{1}=0.003, \gamma=1.4, \gamma_{1}=1.001, \alpha=0.6, \alpha_{0}=.00001, \mu=0.0002, \lambda_{1}=0.0009$ $a=0.6, b=0.4$. From tables $1-3$, we analyze that mean time to system failure, availability and profit decrease with respect to failure rate and value of shape parameter while increases with increase of treatment rate. We analyze that there is a steep change in the values of MTSF, availability and profit with respect to shape parameter. The values of $K_{i}$ for profit function are assumed as $K_{1}=100, K_{0}=500$. Finally, we conclude that our system is more reliable, available and profitable, when random variables have less vale of shape parameters.

\section{REFERENCES}

Barak, A.K., Malik, S. C., \& Barak, M. S. (2015). Reliability analysis of a single-unit system with inspection subject to different weather conditions. Journal of Statistics and Management Systems, 17:2, 195-206, DOI: 10.1080/09720510.2014.914292

Barak, A.K. \& Barak, M.S. (2016). Impact of Abnormal Weather Conditions on Various Reliability Measures of a Repairable System with Inspection. Thailand Statistician, 14(1), 35-45.

Dhankar,A.K. and Malik, S.C.(2011). Cost- benefit analysis of system reliability models with server failure 
during inspection and repair. International Journal of Statistics \& Analysis. 1 (3), 265- 278.

Gupta, R., Kumar, P. and Gupta, A.(2013). Costbenefit analysis of a two dissimilar unit cold standby system with Weibull failure and repair laws. Int. J. Syst. Assur. Eng. Manag. 4(4):327-334.

Kapur, P. K., \& Kapoor, K. R. (1974). A two-unit warm standby system with repair and preventive maintenance. In Indian J. pure appl. Maths . pp. 13-27.

Kishan, R., \& Jain, D. (2014). Classical and Bayesian analysis of reliability characteristics of a two-unit parallel system with Weibull failure and repair laws. International Journal of System Assurance Engineering and Management, 5(3), 252-261.

Kumar A, Saini M, \& Devi K.(2016). Analysis of a redundant system with priority and Weibull distribution for failure and repair. Cogent Mathematics. (just-accepted):1135721.

Kumar,A.; S. K. Chhillar \& S. C. Malik (2016) Analysis of a single-unit system with degradation and maintenance, Journal of Statistics and Management Systems, 19:2, 151-161, DOI: 10.1080/09720510.2014.927609

Kumar, A., Malik, S. C., \& Barak, M. S. (2012). Reliability modelling of a computer system with independent $\mathrm{H} / \mathrm{W}$ and $\mathrm{S} / \mathrm{W}$ failures subject to maximum operation and repair times. International Journal of Mathematical Archive (IJMA). 3(7).

Kumar, A. and Saini, M. (2014). Cost-benefit analysis of a single-unit system with preventive maintenance and Weibull distribution for failure and repair activities, Journal of Applied Mathematics, Statistics and Informatics. 10(2), 5-19.

Kumar, A., \& Malik, S. C. (2012). Reliability modeling of a computer system with priority to $\mathrm{s} / \mathrm{w}$ replacement over $\mathrm{h} / \mathrm{w}$ replacement subject to MOT and MRT. International Journal of Pure and Applied Mathematics, 80(5), 693-709.

Malik, S. C., \& Nandal, P. (2010). Cost-Analysis of Stochastic Models with Priority to Repair Over Preventive Maintenance Subject to Maximum Operation Time, Edited Book, Learning Manual on Modeling, Optimization and Their Applications. Optimization and Their Applications.

Mahmoud, M. A. W., \& Moshref, M. E. (2010). On a two-unit cold standby system considering hardware, human error failures and preventive maintenance.
Mathematical and Computer Modelling, 51(5), 736745.

Malik,S.C. and Bansal, R.K.( 2005) . Profit analysis of single-unit reliability models with repair at different failure modes. Proc. International Conference on Reliability and Safety Engineering, IIT Kharagpur (India), 577-588.

Malik,S.C. (2008). Reliability modeling and profit analysis of a single-unit system with inspection by a server who appears and disappears randomly. Journal of Pure and Applied Mathematika Sciences, LXVII(12), 135-146.

Osaki, S., \& Asakura, T. (1970). A two-unit standby redundant system with repair and preventive maintenance. Journal of Applied Probability, 641-648.

Pawar, D. and Malik,S.C. (2010). Reliability and Economic Measures of a System with Inspection for Online Repair and No Repair Activity in Abnormal Weather. Bulletin of Pure and Applied Sciences, 29 E(2), 355-368. 